\title{
GENERALIZED TAYLOR SERIES AND ORDERS AND TYPES OF ENTIRE FUNCTIONS OF SEVERAL COMPLEX VARIABLES $\left({ }^{1}\right)$
}

\author{
BY \\ FRED GROSS
}

1. Introduction. Entire functions of one complex variable which together with all their derivatives assume integer values at a finite number of integer points have been studied to a considerable extent.

In particular certain lower bounds for the order and type of such transcendental integer valued entire functions have been obtained. Some studies related to this problem can be found in E. G. Straus [1], [2]; T. Schneider [3], S. Kakeya [4], G. Pólya [5]; L. Bieberbach [6], [7]; D. Sato [8] $\left(^{2}\right)$.

For entire functions of $n$ complex variables, order and type are not numbers, but rather certain manifolds in $R^{n}$. The main purpose of this dissertation is to generalize some of the results obtained for entire functions of one complex variable to entire functions of several complex variables. For the sake of simplicity most of our theorems are stated for two variables. The generalizations to more variables are immediate. More specifically we would like to generalize some of the basic results of D. Sato [8] and E. G. Straus [1].

We define a strongly transcendental function as a function $f=\sum a_{i j} z_{1}^{i} z_{2}^{j}$ such that $a_{i j} \neq 0$ for arbitrarily large $i$ and $j$. We also call a transcendental function, which is not strongly transcendental, weakly transcendental.

An entire function $f\left(z_{1}, z_{2}\right)$ will be said to be Hurwitz if all its partial derivatives and its value are integral at the origin.

We shall begin by proving a number of theorems and lemmas and by making some generalized definitions. These will enable us to study order and type points of entire functions in terms of their interpolation series (Generalized Taylor series) at a rectangular array of points.

Two important facts that we shall generalize are the following theorems [8].

I. There exist $2^{\mathrm{x}_{0}}$ entire functions of order $\rho$, type $\sigma$ which together with all their derivatives assume integer values at the points $0,1,2, \cdots, k-1$ for any order $\rho$ with $\rho \geqq k$ or $\rho=k$ and any type $\sigma$ with

Received by the editors October 22, 1962 and, in revised form, November 5, 1964.

(1) Most of this paper is based on the second chapter of a dissertation submitted at University of California, Los Angeles, in July 1962. It was written under the direction of Professor E. G. Straus.

(2) [] : Numbers refer to bibliography. 


$$
\sigma \geqq \sigma_{c}=V(1,2,3, \cdots, k)^{-2 / k}=\left|\begin{array}{ccccc}
1 & 1 & 1 & \cdots & 1 \\
1 & 2 & 3 & \cdots & k \\
1 & 2^{2} & 3^{2} & \ldots & k^{2} \\
\vdots & & & & \\
1 & 2^{k-1} & 3^{k-1} & \ldots & k^{k-1}
\end{array}\right|^{-2 / k}
$$

while there are only denumerably many such functions with $\rho<k$ or $\rho=k$ and $\sigma<\sigma_{c}$.

II. An entire function $f(z)$ satisfying the condition stated in I is a polynomial if $f(z)$ is of order $\rho<k$ or of order $\rho=k$,

$$
\text { type } \sigma<\sigma_{\rho}=\frac{1}{(k-1) !} \prod_{p: \text { prime; } p \leqq k-1} p-[\log (k-1) / \log p]+\frac{1}{p-1}
$$

$(k=1,2,3, \cdots)$.

We shall also characterize order and type curves of entire functions and show that there exists a continuum of entire functions having given order and type and having integral derivatives at a given array of points $\left({ }^{3}\right)$.

\section{Generalized Taylor series and order and type of entire functions.}

LEMMA 2.1. Let $f\left(z_{1}, z_{2}\right)$ be an entire function of two complex variables. Let $\left\{\left(\alpha_{i}, \beta_{j}\right)\right\}_{i, j=1}^{\infty}$ be an infinite rectangular array, $\alpha$ and $\beta$ are complex numbers with $\left\{\alpha_{i}\right\},\left\{\beta_{j}\right\}$ bounded.

Then

$$
f\left(z_{1}, z_{2}\right)=\sum_{i, j=0}^{\infty} \sum_{i j} \prod_{\mu=1}^{i}\left(z_{1}-\alpha_{\mu}\right) \prod_{v=1}^{j}\left(z_{2}-\beta_{v}\right)
$$

where

(2.1) $a_{i j}=-\frac{1}{4 \pi^{2}} \oint_{\left|\xi_{1}\right|=R} \oint_{\left|\xi_{2}\right|=R} f\left(\xi_{1}, \xi_{2}\right) \prod_{\mu=1}^{i+1}\left(\xi_{1}-\alpha_{\mu}\right)^{-1} \prod_{v=1}^{j+1}\left(\xi_{2}-\beta_{v}\right)^{-1} d \xi_{1} d \xi_{2}$ where $R>\sup _{i=1,2, \cdots}\left\{\left|\alpha_{i}\right|,\left|\beta_{i}\right|\right\}$.

Proof. We apply Sato's theorem twice. For $z_{2}$ fixed we have:

(3) After the completion of this paper it was brought to the author's attention that some of the results on order and type curves appearing in this paper had been published in Russian by B. A. Fuks. More recently his work has been translated into English [9]. The author's presentation is less general than that of Fuks and consequently more readable. Fuks does not deal with any of the questions related to entire functions with integral derivatives at certain points having prescribed order and type. 
where

$$
f\left(z_{1}, z_{2}\right)=F\left(z_{1}\right)=\sum_{i=1}^{\infty} \phi_{i}\left(z_{2}\right) \prod_{\mu=1}^{i}\left(z_{1}-\alpha_{\mu}\right)
$$

$$
\phi_{i}\left(z_{2}\right)=\frac{1}{2 \pi i} \oint_{\left|\xi_{1}\right|=R} \frac{f\left(\xi_{1}, z_{2}\right) d \xi_{1}}{\left(\xi_{1}-\alpha_{1}\right) \cdots\left(\xi_{1}-\alpha_{i+1}\right)} .
$$

On the other hand,

where

$$
\phi_{i}\left(z_{2}\right)=\sum_{j=0}^{\infty} a_{i j} \prod_{v=1}^{j}\left(z_{2}-\beta_{v}\right)
$$

$$
a_{i j}=\frac{1}{2 \pi i} \oint_{\left|\xi_{2}\right|=R} \frac{\phi_{1}\left(\xi_{2}\right) d \xi_{2}}{\left(\xi_{2}-\beta_{1}\right) \cdots\left(\xi_{2}-\beta_{j+1}\right)} .
$$

Note: The condition that the coordinates of the points of the rectangular array be bounded can be shown to be necessary if the expansion is to converge for all entire functions.

Definition 2.1. Let $f\left(z_{1}, \cdots, z_{k}\right)$ be an entire function. The set of all $k$-tuples $\left(\rho_{1}, \cdots, \rho_{k}\right)$, where $\rho_{i} \geqq 0$ for $i=1, \cdots, k$ such that

$$
M\left(r_{1}, \cdots, r_{k}\right) / \exp \left[-r_{1}^{\rho_{1}+\varepsilon}+\cdots+r_{k}^{\rho_{k}+\varepsilon}\right]
$$

is bounded for every $\varepsilon>0$ and

$$
M\left(r_{1}, \cdots, r_{k}\right) / \exp \left[r_{1}^{\rho_{1}}+\cdots+r_{i}^{\rho_{i}-\varepsilon}+\cdots+r_{k}^{\rho_{k}}\right]
$$

is unbounded for each $i=1, \cdots, k$ and for every $\varepsilon>0$ is called the order of $f$ and is denoted by $\rho\left(\rho_{1}, \rho_{2}, \cdots, \rho_{k}\right)$. Each point $\left(\rho_{1}, \cdots, \rho_{k}\right)$ is called an order point of $f$.

Definition 2.2. Let $f\left(z_{1}, \cdots, z_{k}\right)$ be an entire function and let $\left(\rho_{1}, \cdots, \rho_{k}\right)$ be an order point of $f$. The set of all $k$-tuples $\left(\sigma_{1}, \cdots, \sigma_{k}\right)$ such that

$$
M\left(r_{1}, \cdots, r_{k}\right) / \exp \left[\left(\sigma_{1}+\varepsilon\right) r_{1}^{\rho_{1}}+\cdots+\left(\sigma_{k}+\varepsilon\right) r_{k}^{\rho_{k}}\right]
$$

is bounded for every $\varepsilon>0$ and

$$
M\left(r_{1}, \cdots, r_{k}\right) / \exp \left[\sigma_{1} r_{1}^{\rho_{1}}+\cdots+\left(\sigma_{i}-\varepsilon\right) r_{i}^{\rho_{i}}+\cdots+\sigma_{k} r_{k}^{\rho_{k}}\right]
$$

is unbounded for $i=1, \cdots, k$ and for every $\varepsilon>0$ is called the type of $f$ at $\left(\rho_{1}, \cdots, \rho_{k}\right)$, denoted by $\sigma_{\rho_{1} \cdots \rho_{k}}\left(\sigma_{1}, \cdots, \sigma_{k}\right)$.

In analogy to the theory for functions of one variable we have here

LEMMA 2.2. Let

$$
f=\sum a_{n_{1} n_{2}} z_{1}^{n_{1}} z_{2}^{n_{2}}
$$

If

$$
M\left(r_{1}, r_{2}\right)<\exp \left[r_{1}^{\rho_{1}+\varepsilon}+r_{2}^{\rho_{2}+\varepsilon}\right]
$$


for sufficiently large $r_{1}$ or $r_{2}$ for every $\varepsilon>0$, then

$$
\left|a_{n_{1} n_{2}}\right|<\left(\frac{e\left(\rho_{1}+\varepsilon\right)}{n_{1}}\right)^{n_{1} /\left(\rho_{1}+\varepsilon\right)}\left(\frac{e\left(\rho_{2}+\varepsilon\right)}{n_{2}}\right)^{n_{2} /\left(\rho_{2}+\varepsilon\right)}
$$

for $n_{1}+n_{2}$ sufficiently large for every $\varepsilon>0$, and conversely; $\rho_{1}$ and $\rho_{2}$ are real numbers.

For the proof which is entirely analogous to the one-variable case see e.g. [8]. This estimate generalizes to the generalized Taylor series of Lemma 2.1.

LEMMA 2.3. Let

$$
f\left(z_{1}, z_{2}\right)=\sum_{k, l=0}^{\infty} \sum_{k l} \prod_{i=1}^{k}\left(z_{1}-\alpha_{i}\right) \prod_{j=1}^{l}\left(z_{2}-\beta_{j}\right),
$$

where $\left\{\alpha_{i}\right\},\left\{\beta_{j}\right\}$ are bounded.

If

$$
M\left(R_{1}, R_{2}\right)<\exp \left[R_{1}^{\rho_{1}+\varepsilon}+R_{2}^{\rho_{2}+\varepsilon}\right]
$$

for every $\varepsilon>0$ for sufficiently large $R_{1}$ or $R_{2}$, then

$$
\left|a_{n_{1} n_{2}}\right|<\left(\frac{e \rho_{1}+\varepsilon}{n_{1}}\right)^{n_{1} /\left(\rho_{1}+\varepsilon\right)}\left(\frac{e \rho_{2}+\varepsilon}{n_{2}}\right)^{n_{2} /\left(\rho_{2}+\varepsilon\right)} \text { for every } \varepsilon>0
$$

for $n_{1}+n_{2}$ sufficiently large and conversely.

The corresponding results for the type follow in a similar manner.

LEMMA 2.4. Let $f\left(z_{1}, z_{2}\right)$ be as in Lemma 2.3. If

$$
M\left(R_{1}, R_{2}\right)<\exp \left[\left(\sigma_{1}+\varepsilon\right) R_{1}^{\rho_{1}}+\left(\sigma_{2}+\varepsilon\right) R_{2}^{\rho_{2}}\right]
$$

for sufficiently large $R_{1}$ or $R_{2}$ for every $\varepsilon>0$, then

$$
\left|a_{n_{1} n_{2}}\right|<\left(\frac{e \rho_{1}\left(\sigma_{1}+\varepsilon\right)}{n_{1}}\right)^{n_{1} / \rho_{1}}\left(\frac{e \rho_{2}\left(\sigma_{2}+\varepsilon\right)}{n_{2}}\right)^{n_{2} / \rho_{2}}
$$

for $n_{1}+n_{2}$ sufficiently large for every $\varepsilon>0$ and conversely.

\section{Hurwitz functions.}

THEOREM 3.1. Let $f\left(z_{1}, z_{2}\right)$ be a Hurwitz function (4). If $\rho_{1}<1$ and $\rho_{2}<1$ for any order point $\left(\rho_{1}, \rho_{2}\right)$ of $f$, then $f$ is a polynomial.

Proof.

$$
\frac{\left|a_{n_{1} n_{2}}\right|}{n_{1} ! n_{2} !}<\left(\frac{e \rho_{1}+\varepsilon}{n_{1}}\right)^{n_{1} /\left(\rho_{1}+\varepsilon\right)}\left(\frac{e \rho_{2}+\varepsilon}{n_{2}}\right)^{n_{2} /\left(\rho_{2}+\varepsilon\right)}<n_{1}^{-n_{1} /\left(\rho_{1}+\varepsilon^{\prime}\right)} n_{2}^{-n_{2} /\left(\rho_{2}+\varepsilon^{\prime}\right)}
$$

(4) In this section all results remain valid if we interpret "integer" or "Gaussian integer" to mean integer in an imaginary quadratic field. 
for sufficiently large $n_{1}+n_{2}$. Thus we get

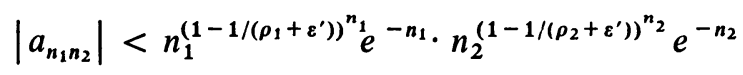

$\rho_{i}<1, \rho_{i}+\varepsilon<1$ or $\left(1-1 /\left(\rho_{i}+\varepsilon\right)\right)<0$ for sufficiently small $\varepsilon>0$ and for $i=1,2$. Thus $a_{n_{1} n_{2}}=0$ for sufficiently large $n_{1}+n_{2}$.

THEOREM 3.2. Let $f\left(z_{1}, z_{2}\right)$ be a Hurwitz function with an order point $\left(\rho_{1}, \rho_{2}\right)$. If $\rho_{1}=1, \rho_{2}=1, \sigma_{1}<1$ and $\sigma_{2}<1$ for some $\left(\sigma_{1}, \sigma_{2}\right) \in \sigma_{\rho_{1} \rho_{2}}$ then $f$ is a polynomial.

\section{Proof.}

$$
\begin{aligned}
\left|a_{n_{1} n_{2}}\right| & <\left(\frac{e\left(\sigma_{1}+\varepsilon\right)}{n_{1}}\right)^{n_{1}}\left(\frac{e\left(\sigma_{2}+\varepsilon\right)}{n_{2}}\right)^{n_{1}} n_{1} ! n_{2} ! \\
& <\left(\frac{e\left(\sigma_{1}+\varepsilon^{\prime}\right)}{n_{1}}\right)^{n_{1}}\left(\frac{e\left(\sigma_{2}+\varepsilon^{\prime}\right)}{n_{2}}\right)^{n_{2}} n_{1}^{n_{1}} n_{2}^{n_{2}} e^{-n_{1}} e^{-n_{2}}
\end{aligned}
$$

for sufficiently large $n_{1}+n_{2}$ and $\varepsilon^{\prime}>\varepsilon$ or

or

$$
\left|a_{n_{1} n_{2}}\right|<\left(\sigma_{1}+\varepsilon^{\prime}\right)^{n_{1}}\left(\sigma_{2}+\varepsilon^{\prime}\right)^{n_{2}}<1
$$

$$
\left|a_{n_{1} n_{2}}\right|=0
$$

for $n_{1}+n_{2}$ sufficiently large.

THEOREM 3.3. There exist $2^{\aleph_{0}}$ strongly transcendental entire functions with order point $\left(\rho_{1}, \rho_{2}\right)$ and type point $\left(\sigma_{1}, \sigma_{2}\right)$ which together with all their derivatives assume Gaussian integer values at the points $\left(\alpha_{i}, \beta_{j}\right)(i=1, \cdots, \bar{k} ; j=1, \cdots, k$ with $k \leqq k$, and $\alpha$ and $\beta$ are complex numbers) for every $\left(\rho_{1}, \rho_{2}\right)$ and $\left(\sigma_{1}, \sigma_{2}\right)$ such that $\rho_{1} \geqq k$ and $\rho_{2} \geqq k$ or such that $\rho_{1}=\bar{k}, \rho_{2}=k, \sigma_{1}>\sigma_{c 1}$ and $\sigma_{2}>\sigma_{c 2}$. Here

$$
\sigma_{c 1}=\left|V\left[\alpha_{i}\right]\right|^{-2 / k} \text { and } \sigma_{c 2}=\left|V\left[\beta_{i}\right]\right|^{-2 / k},
$$

with $V$ denoting the Vandermonde.

Proof. Set

$$
f\left(z_{1}, z_{2}\right)=\sum_{s=0}^{\infty} \sum_{t=0}^{\infty} a_{s t}\left(z_{1}-\alpha_{1}\right)^{s_{1}}\left(z_{2}-\alpha_{2}\right)^{s_{2}} \cdots\left(z_{1}-\alpha_{k}\right)^{s_{k}} \cdot\left(z_{2}-\beta_{1}\right)^{t_{1}} \cdots\left(z_{2}-\beta_{k}\right)^{t_{k}}
$$

where

$$
s_{1}+s_{2}+\cdots+s_{k}=s \text { and } t_{1}+t_{2}+\cdots+t_{k}=t
$$

are chosen such that the terms

$$
s_{l} !\left|\left(\alpha_{l}-\alpha_{1}\right) \cdots\left(\alpha_{l}-\alpha_{l-1}\right)\left(\alpha_{l+1}-\alpha_{l}\right) \cdots\left(\alpha_{k}-\alpha_{l}\right)\right|^{s_{l}}=s_{l} ! \gamma_{1 l}^{s_{l}}
$$


are roughly of the same order for all $l$. Similarly

$$
t_{l} !\left|\left(\beta_{l}-\beta_{1}\right)\left(\beta_{l}-\beta_{2}\right) \cdots\left(\beta_{l}-\beta_{l-1}\right)\left(\beta_{l+1}-\beta_{l}\right)\left(\beta_{l+2}-\beta_{l}\right) \cdots\left(\beta_{k}-\beta_{l}\right)\right|^{t_{l}}=t_{l} ! \gamma_{2 l}^{t_{l}}
$$

is chosen this way also.

More precisely we set

$$
s_{l} ! \gamma_{1 l}^{s_{l}}=\Gamma\left(\frac{s}{k}\right) \gamma_{1}^{s / k} \cdot o\left(e^{e s}\right), \quad \gamma_{1}=\left|V\left(\alpha_{i}\right)\right|^{2 / k}
$$

and

$$
t_{l} ! \gamma_{2 l}^{t_{l}}=\Gamma\left(\frac{t}{k}\right) \gamma_{2}^{t / k} \cdot o\left(e^{\varepsilon t}\right), \quad \gamma_{2}=\left|V\left(\beta_{j}\right)\right|^{2 / k}
$$

Then

$$
\begin{array}{ll}
s_{l}=\frac{s}{k}+c_{l} \frac{s}{\log s}+o\left(\frac{s}{\log s}\right), & c_{l}=\log \frac{\left(\gamma_{1} / \gamma_{1 l}\right)}{k}, \\
t_{l}=\frac{t}{k}+c_{l}^{\prime} \frac{t}{\log t}+o\left(\frac{t}{\log t}\right), & c_{l}^{\prime}=\frac{\log \left(\gamma_{2} / \gamma_{2 l}\right)}{k} .
\end{array}
$$

Since $\Sigma c_{l}=\sum c_{l}^{\prime}=0$ we can choose the $o(s / \log s)$ and $o(t / \log t)$ error terms so as to satisfy (3.1).

Now let

and

$$
a_{s(t+1)}=a_{s\left(t_{1}+\cdots+\left(t_{l}+1\right)+\cdots+t_{k}\right)}
$$

$$
a_{(s+1) t}=a_{\left(s_{1}+\cdots+\left(s_{h}+1\right)+\cdots+s_{k}\right) t} \cdot
$$

Then $a_{s t}$ is the last term which enters into the computation of

$$
\frac{\partial^{s_{h}+t_{l}} f\left(\alpha_{h}, \beta_{l}\right)}{\partial z_{1}^{s_{h}} \partial z_{2}^{t_{l}}}
$$

In other words $a_{p q}$ with $p>s$ or $q>t$ makes no contribution to this derivative since its multiplier vanishes at $\left(\alpha_{h}, \beta_{l}\right)$.

We have

If we choose

$$
\begin{aligned}
& \frac{\partial^{s_{h}+t_{l}} f\left(\alpha_{h}, \beta_{l}\right)}{\partial z_{1}^{s_{\lambda} \partial z_{2}^{t_{l}}}}=\left(\text { contribution of terms preceding } a_{s t}\right) \\
& +a_{s t} s_{h} ! t_{l} ! \prod_{\mu=1 ; \mu \neq h}^{k}\left(\alpha_{h}-\alpha_{\mu}\right)^{s_{\mu}} \prod_{v=1 ; v \neq l}^{k}\left(\beta-\beta_{v}\right)^{t_{v}} .
\end{aligned}
$$

$$
\frac{\partial^{s_{h}+t_{l}} f\left(\alpha_{h}, \beta_{l}\right)}{\partial z_{1}^{s_{h}} \partial z_{2}^{t_{l}}}
$$

as a Gaussian integer sufficiently near to the first term on the right we have (for fixed $\left.c \geqq(2)^{-1 / 2}\right)\left({ }^{5}\right)$.

(5) This constant obviously depends on the number field. 
where

$$
\begin{aligned}
\left|a_{s t}\right| & \leqq c \mid\left[s_{h} ! t_{l} ! \prod_{\mu=1 ; \mu \neq h}^{k}\left(\alpha_{h}-\alpha_{\mu}\right)^{s_{\mu}} \prod_{v=1 ; v \neq l}^{k}\left(\beta_{l}-\beta_{v}\right)^{t_{v}}\right]^{-1} \\
& =c \mid\left[\left.s_{h} ! \gamma_{\alpha h}^{s / k} \cdot o(\exp (\varepsilon s)) t_{l} ! \gamma_{\beta l}^{t / k} \cdot o(\exp (\varepsilon t))\right|^{-1}\right.
\end{aligned}
$$

Thus

$$
\gamma_{r p}=\prod_{u=1 ; u \neq p}^{N_{p}}\left(r_{p}-r_{u}\right) .
$$

But

$$
\left|a_{s t}\right| \leqq c\left|\left[\Gamma\left(\frac{s}{k}\right) \gamma_{1}^{s / k} \cdot o\left(e^{e s}\right) \cdot \Gamma\left(\frac{t}{k}\right) \gamma_{2}^{t / k} \cdot o\left(e^{e t}\right)\right]\right|^{-1}
$$

$$
\Gamma\left(\frac{s}{k}\right) \gamma_{1}^{s / k} \cdot o\left(e^{e s}\right)<\left(\frac{e k+\varepsilon}{s}\right)^{s /(k+\varepsilon)}
$$

for sufficiently large $s$. This follows from the fact

$$
\left[\left(\frac{s}{k}\right)^{s / k} e^{-s / k} \gamma_{1}^{s / k} \cdot o\left(e^{e s}\right)\right]^{-1}=\left(\frac{s \gamma_{1}}{e \bar{k}}\right)^{-s / k} o\left(e^{e s}\right)^{-1}
$$

(or since $o\left(e^{e s}\right)>1$, which may be assumed)

$$
<\left(\frac{s \gamma_{1}}{e k}\right)^{-s / k}<s^{-s /(k+\varepsilon)}
$$

for arbitrarily small $\varepsilon>0$ and for sufficiently large $s$. Similarly

$$
\Gamma\left(\frac{t}{k}\right) \gamma_{2}^{t / k} \cdot o\left(e^{e t}\right)<\left(\frac{e k+\varepsilon}{t}\right)^{t /(k+\varepsilon)}
$$

for arbitrarily small $\varepsilon>0$ and for sufficiently large $t$. Thus

$$
\left|a_{s t}\right|<\left(\frac{e \hat{k}+\varepsilon}{s}\right)^{s /(k+\varepsilon)}\left(\frac{e k+\varepsilon}{t}\right)^{t /(k+\varepsilon)}
$$

for arbitrarily small $\varepsilon>0$ and for sufficiently large $s+t$. Hence by Lemma 2.3

$$
M\left(R_{1}, R_{2}\right)<\exp \left[R_{1}^{k+\varepsilon}+R_{2}^{k+\varepsilon}\right] .
$$

Similarly for

$$
\left(\rho_{1}, \rho_{2}\right)=(k, k)
$$

we know that

Thus

$$
\left|a_{s t}\right|<\left(\frac{e k \gamma_{1}^{-1}}{s}\right)^{s / k}\left(\frac{e k \gamma_{2}^{-1}}{t}\right)^{t / k}
$$

$$
\sigma_{1} \leqq \gamma_{1}^{-1} \text { and } \sigma_{2} \leqq \gamma_{2}^{-1} \text {. }
$$

Clearly if we pick $c$ sufficiently large then the inequality (3.4) does not determine 


$$
\frac{\partial^{s_{n}+t_{l}} f\left(\alpha_{h}, \beta_{l}\right)}{\partial z_{1}^{s} \frac{z_{2}}{\partial z_{2} l}}
$$

uniquely and our construction leads to a continuum of functions satisfying the conditions of our theorem.

THEOREM 3.4. There exists at most a denumerable number of $f$ with an order point $(k, k)$, where $f$ is of the kind described in Theorem 3.3 and such that a type point $\left(\sigma_{1}, \sigma_{2}\right)$ which is in $\sigma_{k k}$ satisfies

$$
\sigma_{1}<\left|V\left[\alpha_{i}\right]\right|^{-2 / k} \text { and } \sigma_{2}<\left|V\left[\beta_{i}\right]\right|^{-2 / k} \text {. }
$$

Proof. It suffices to show that under these conditions

$$
\left|a_{s t} s_{h} ! t_{l} ! \prod_{i \neq h}\left(\alpha_{h}-\alpha_{i}\right)^{s_{i}} \prod_{j \neq l}\left(\beta_{l}-\beta_{j}\right)^{t_{j}}\right|<1 / 2
$$

for sufficiently large $s$ and $t$, since that makes the determination of $a_{s t}$ so that $\partial^{s_{h}+t_{l}} f\left(\alpha_{h}, \beta_{l}\right) / \partial z_{1}^{s_{h}} \partial z_{2}^{t_{l}}$ is a Gaussian integer unique.

Equation (3.5) implies

$$
\left.\left|a_{s t}(s / \bar{k})^{s / k}(t / k)^{t / k}\right| \gamma_{1}\right|^{s / k}\left|\gamma_{2}\right|^{t / k} o\left(e^{\varepsilon s}\right) o\left(e^{e t}\right) \exp [-(s / k+t / k)] \mid<1 / 2 .
$$

But

and

$$
\left|a_{s t}\right|<\left(\frac{e \hat{k}\left(\sigma_{1}+\varepsilon\right)}{s}\right)^{s / k}\left(\frac{e k\left(\sigma_{2}+\varepsilon\right)}{t}\right)^{t / k}
$$

$$
\left.\left|\left(\frac{e \bar{k}\left(\sigma_{1}+\varepsilon\right)}{s}\right)^{s / k}(s / \bar{k})^{s / k} e^{-s / k}\right| \gamma_{1}\right|^{s / k} o\left(e^{\varepsilon s}\right)|=|\left[\left(\sigma_{1}+\varepsilon\right)\left|\gamma_{1}\right|\right]^{s / \bar{k}} o\left(e^{\varepsilon s}\right) \mid
$$

If $\left(\sigma_{1}+\varepsilon\right)\left|\gamma_{1}\right|<1$, then $\left[\left(\sigma_{1}+\varepsilon\right)\left|\gamma_{1}\right|\right]^{s / k} o\left(e^{e s}\right)<1 / 2$ for sufficiently large $s$. Similarly for $\left(\sigma_{2}+\varepsilon\right)\left|\gamma_{2}\right|<1$ we get $\left[(\sigma+\varepsilon)\left|\gamma_{2}\right|\right]^{t / k} o\left(e^{e t}\right)<1 / 2$ for sufficiently large $t$ and our theorem follows.

Our results so far have been connected to functions integral valued at a rectangular array of points. We now wish to show that any finite set of Gaussian integers can be transformed into a rectangular array of points by one-to-one algebraic transformations and that the arithmetical restrictions can be essentially preserved by these transformations.

We are going to consider transformations of the group, $G$, generated by transformations of the types

$$
y_{1}=c_{1} x_{1}+f\left(x_{2}\right), \quad y_{2}=c_{2} x_{2}, c_{1} c_{2} \neq 0
$$

and

$$
y_{1}=c_{1} x_{1}, y_{2}=c_{2} x_{2}+g\left(x_{1}\right) \text {, }
$$


$c_{1} c_{2} \neq 0$ where $f, g$ are polynomials. The inverses are obviously of the same form. It is easy to show (Theorem 3.5) that any $n$-tuple of points $\left(a_{i}, b_{i}\right) ; i=1, \cdots, n$ can be transformed into any other $n$-tuple $\left(c_{i}, d_{i}\right) ; i=1, \cdots, n$ by a transformation of $G$. Since we are interested in transformations which map the lattice points with Gaussian integral coordinates into themselves and a function with Gaussian integral partial derivatives at certain lattice points into a function with the same property at the image points, we shall be concerned with the subgroup $G_{1}$ of $G$ generated by those transformations (3.6) and (3.7) for which $c_{1}, c_{2}$ are Gaussian units and the polynomials $f, g$ together with all their derivatives are Gaussian integral valued (it suffices to consider $f, h$ with Gaussian integral coefficients). For every $n$-tuple of lattice points $\left(a_{i}, b_{i}\right) ; i=1, \cdots, n$ there exists a transformamation of $G_{1}$ which maps it into a rectangular array of lattice points $\left(c_{j}, d_{k}\right) ; j=1, \cdots, l ; k=1, \cdots, m ; l m=n$.

LEMMA 3.1. Let $\left(\alpha_{1}, \beta_{1}\right), \cdots,\left(\alpha_{k}, \beta_{k}\right)$ be a set of pairs of complex numbers. There exists a transformation given by $y_{1}=x_{1}$ and $y_{2}=A x_{1}+x_{2}$ which maps $\left\{\left(\alpha_{i}, \beta_{i}\right)\right\}, i=1, \cdots, k$, into some set of pairs $\left\{\left(\alpha_{i}, \beta_{i}^{\prime}\right)\right\}, i=1, \cdots, k$, so that $A$ is a Gaussian integer and $\beta_{i}^{\prime} \neq \beta_{j}^{\prime}$ for $i \neq j$.

Proof. Pick $A$ a Gaussian integer so that $A \neq\left(\alpha_{s}-\alpha_{t}\right) /\left(\beta_{t}-\beta_{s}\right)$ for any pair of distinct indices $s, t$. Then $A \alpha_{s}+\beta_{s} \neq A \alpha_{t}+\beta_{t}$ for $s \neq t$.

THEOREM 3.5. Given two n-tuples of pairs of complex numbers $\left(a_{j}, b_{j}\right)$ and $\left(c_{j}, d_{j}\right) ; j=1, \cdots, n$. There exists a transformation $T \in G$ so that $T$ maps $\left(a_{j}, b_{j}\right)$ into $\left(c_{j}, d_{j}\right)$.

Proof. By the above Lemma we can map $\left(a_{j}, b_{j}\right)$ into $\left(a_{j}^{\prime}, b_{j}\right)$, where the $a_{j}^{\prime}$ are distinct for $j=1, \cdots, n$. The transformation $y_{2}=x_{2}, y_{1}=x_{1}+$ $\sum_{i=1}^{n}\left(c_{i}-a_{i}^{\prime}\right) \prod_{j \neq i}\left(x_{2}-a_{j}^{\prime}\right) / \prod_{j \neq i}\left(a_{t}^{\prime}-a_{j}^{\prime}\right)$ maps $\left(a_{j}^{\prime}, b_{j}\right)$ into $\left(c_{j}, b_{j}\right)$. Again applying the above Lemma we can map this into $\left(c_{j}, b_{j}^{\prime}\right)$, where the $b_{j}^{\prime}$ are distinct for $j=1, \cdots, n$. The transformation

$$
y_{1}=x_{1}, y_{2}=x_{2}+\sum_{i=1}^{n}\left(d_{i}-b_{i}^{\prime}\right) \prod_{j \neq i}\left(x_{1}-b_{j}^{\prime}\right) / \prod_{j \neq i}\left(b_{i}^{\prime}-b_{j}^{\prime}\right)
$$

yields the desired result.

LEMMA 3.2. Let $\phi\left(x_{1}, x_{2}\right)$ be an entire function in two complex variables whose value and derivatives are Gaussian integers at the pairs of Gaussian integers $\left(a_{1}, b_{1}\right), \cdots,\left(a_{k}, b_{k}\right)$. Let $y_{1}=c x_{1}+f\left(x_{2}\right)$ and $y_{2}=x_{2}$ be a map which takes $\left(a_{i}, b_{i}\right)$ into $\left(\alpha_{i}, b_{i}\right)$ for $i=1, \cdots, k$, where $c$ is a Gaussian unit and $f\left(x_{2}\right)$ is a polynomial with Gaussian integral coefficients. In such a case all the derivatives as well as the value of $F\left(y_{1}, y_{2}\right)=\phi\left(x_{1}, x_{2}\right)$, considered as a function of $y_{1}$ and $y_{2}$, are Gaussian integers at $\left(\alpha_{1}, b_{1}\right), \cdots,\left(\alpha_{k}, b_{k}\right)$. 
Proof. $\partial^{m+n} F\left(\alpha_{i}, b_{i}\right) / \partial y_{1}^{m} \partial y_{2}^{n}$ can be expressed as a linear combination with integral coefficients of terms $\partial^{p+q} \phi\left(a_{i}, b_{i}\right) / \partial x_{1}^{p} \partial x_{2}^{q}$ multiplied by derivatives of $x_{1}$ and $x_{2}$ with respect to $y_{1}$ and $y_{2}$. Since all these quantities are Gaussian integers the result follows.

By virtue of Lemmas 3.5 and 3.6 and Theorem 3.5 it suffices to consider rectangular arrays in place of general ones when dealing with certain questions. With this is mind, we prove the following theorem.

THEOREM 3.6. Let $f\left(z_{1}, z_{2}\right)$ be an entire function of order $\rho\left(\rho_{1}, \rho_{2}\right)$. Let $(k, \bar{k})$ be an order point of $f$. Suppose that the values of $f$ and its derivatives are Gaussian integers at the rectangular array of points

$$
\left(\alpha_{i}, \beta_{j}\right) ; i=0, \cdots, \bar{k}-1 ; j=0, \cdots, k-1,
$$

where $\alpha_{i}$ and $\beta_{j}$ are Gaussian integers. If $\left(\sigma_{1}, \sigma_{2}\right)$ is a type point of $f$ at $(\bar{k}, k)$ such that

$$
\sigma_{j}<\left|V_{j}\right|^{-1} \prod_{p<m_{j} ; p: \text { prime }} p^{-\left(\left[\log m_{j} / \log p\right]-1 /(p-1)\right)} ; j=1,2,
$$

then $f$ is a polynomial.

Here $V_{1}=V\left[\alpha_{i}\right], V_{2}=V\left[\beta_{i}\right]$ with $V$ denoting the Vandermonde, $m_{1}=\max _{i, j}\left\{\left|\alpha_{i}-\alpha_{j}\right|\right\}$ and $m_{2}=\max _{i, j}\left\{\left|\beta_{i}-\beta_{j}\right|\right\}$.

Proof.

$$
\begin{aligned}
f\left(z_{1}, z_{2}\right)= & \sum_{s=0}^{\infty} \sum_{l=0}^{k-1} \sum_{t=0}^{\infty} \sum_{h=0}^{k-1} a_{(s k+l)(t k+h)}\left(z_{1}-\alpha_{0}\right)^{s+1} \cdots\left(z_{1}-\alpha_{l-1}\right)^{s+1} \\
& \cdot\left(z_{1}-\alpha_{l}\right)^{s} \cdots\left(z_{1}-\alpha_{k-1}\right)^{s}\left(z_{2}-\beta_{0}\right)^{t+1} \cdots\left(z_{2}-\beta_{k-1}\right)^{t}
\end{aligned}
$$

with

$$
\begin{aligned}
& a_{(s k+l)(t k+h)}=\frac{1}{4 \pi^{2}} \oint_{\left|\xi_{1}\right|=R_{1}} \oint_{\left|\xi_{2}\right|=R_{2}}
\end{aligned}
$$

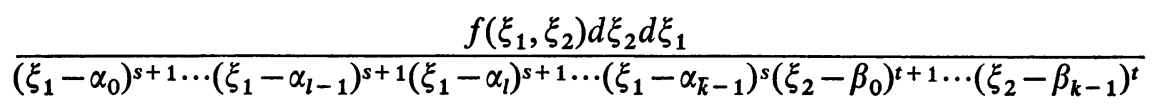

where

If

$$
R_{1}>\max _{i}\left\{\left|\alpha_{i}\right|\right\} \text { and } R_{2}>\max _{i}\left\{\left|\beta_{i}\right|\right\} \text { and } R_{1} \neq \infty \neq R_{2} \text {. }
$$

then

$$
\left|f\left(z_{1}, z_{2}\right)\right|<c \exp \left[\left(\sigma_{1}+\varepsilon\right) R_{1}^{k}+\left(\sigma_{2}+\varepsilon\right) R_{2}^{k}\right]
$$

$$
\begin{gathered}
\left|a_{(s k+l)(t k+h)}\right|<\frac{1}{4 \pi^{2}} \cdot \frac{4 \pi^{2} R_{1} R_{2}}{R_{1}^{s} \overline{k+l+1} R_{2}^{t k+h+1}} \\
\cdot \frac{c \exp \left[\left(\sigma_{1}+\varepsilon\right) R_{1}^{k}+\left(\sigma_{2}+\varepsilon\right) R_{2}^{k}\right]}{\left|\left(1-\frac{\alpha_{0}}{R_{1}}\right)^{s+1} \cdots\left(1-\frac{\alpha_{l}}{R_{1}}\right)^{s+1}\left(1-\frac{\alpha_{l+1}}{R_{1}}\right)^{s} \cdots\left(1-\frac{\alpha_{k-1}}{R_{1}}\right)^{s}\left(1-\frac{\beta_{0}}{R_{2}}\right)^{t+1}\left(1-\frac{\beta_{k-1}}{R_{2}}\right)^{t}\right|} .
\end{gathered}
$$


Clearly

while

$$
\left|\left(1-\frac{\alpha_{l}}{R_{1}}\right)^{R_{1}}\right|=\left|\exp \left[-\alpha_{l}+o\left(1 / R_{1}\right)\right]\right|
$$

$$
\left|\left(1-\frac{\beta_{h}}{R_{2}}\right)\right|=\left|\exp \left[-\beta_{h}+o\left(1 / R_{2}\right)\right]\right|
$$

and both are $>\gamma^{-1}$ for some $\gamma$. Thus

$$
\left|a_{(s k+l)(t k+h)}\right|<\frac{\exp \left[\left(\sigma_{1}+\varepsilon\right) R_{1}^{k}+\left(\sigma_{2}+\varepsilon\right) R_{2}^{k}\right]}{R_{1}^{s k} R_{2}^{t k}} \cdot \frac{c \gamma^{(s k+l+1) / R_{1}+(t k+h+1) / R_{2}}}{R_{1}^{l+1} R_{2}^{h+1}} .
$$

We now minimize

$$
\frac{\exp \left[\left(\sigma_{1}+\varepsilon\right) R_{1}^{k}+\left(\sigma_{2}+\varepsilon\right) R_{2}^{k}\right.}{R_{1}^{s k} R_{2}^{t k}}
$$

and get

$$
R_{1}=\left(\frac{s}{\sigma_{1}+\varepsilon}\right)^{1 / k} \text { and } R_{2}=\left(\frac{t}{\sigma_{2}+\varepsilon}\right)^{1 / k}
$$

Thus

$$
\left|a_{(s \bar{k}+l)(t k+h)}\right|<e^{(s+t)} \gamma_{01}^{s^{(1-1 / \bar{k})}} \gamma_{02}^{t^{(1-1 / k)}} /\left(\frac{s}{\sigma_{1}+\varepsilon}\right)^{s}\left(\frac{t}{\sigma_{2}+\varepsilon}\right)^{t}
$$

for some $\gamma_{01}$ and $\gamma_{02}$. Hence

$$
\left|a_{(s k+l)(t k+h)}\right|<\left[\left(e \sigma_{1}+\varepsilon_{1}\right)^{s} s^{-s}\right]\left[\left(e \sigma+\varepsilon_{2}\right)^{t} t^{-t}\right]
$$

for sufficiently large $s+t$ and for $\varepsilon_{1}>0$ and $\varepsilon_{2}>0$.

We introduce the following notation: $r_{1}=s, r_{2}=t, \gamma_{1 j}=\alpha_{j}, \gamma_{2 j}=\beta_{j}, \varepsilon_{1}=l$, $\varepsilon_{2}=h, \quad \delta_{1}=k-1, \quad \delta_{2}=k-1, \quad \eta_{1 j}=x_{j}, \quad \eta_{2 j}=y_{j}, \quad \eta_{1}=m, \quad \eta_{2}=n$, and $s(x, k)=\left\{\right.$ non-negative integers $\left.x_{i} ; \sum_{i=0}^{\delta_{i}} x_{i}=x\right\}$.

Let

and let

$$
\psi=\prod_{i=1}^{2} \prod_{j=0}^{\varepsilon_{i}}\left(\xi_{i}-\gamma_{i j}\right)^{r_{i}+1} \cdot \prod_{j=\varepsilon_{i}+1}^{\delta_{i}}\left(\xi_{i}-\gamma_{i j}\right)^{r_{i}}
$$

$$
\begin{aligned}
\theta_{m}= & {\left[\sum_{s(x, \bar{k}), x_{m}=0 ; s(y, k), y_{n}=0} x ! y ! \prod_{i=1}^{2} \prod_{j=0}^{\delta_{i}}\left(\eta_{i j} !\right)^{-1} \cdot(-1)^{x+y}\right.} \\
& \cdot \prod_{i=1}^{2} \prod_{j=0}^{\varepsilon_{i}}\left(r_{i}+\eta_{i j}\right) ! \cdot \prod_{j=\varepsilon_{i}+1}^{\delta_{i}}\left(r_{i}-1+\eta_{i j}\right) ! \\
& \left.\cdot\left(\prod_{i=1}^{2}\left(r_{i} !\right)^{\varepsilon_{i}+1}\left(\left(r_{i}-1\right) !\right)^{\delta_{i}-\varepsilon_{i}}\right)^{-1}\right] \\
& \cdot\left[\prod_{i=1}^{2} \prod_{j=0}^{\varepsilon_{i}}\left(\gamma_{i n_{i}}-\gamma_{i j}\right)^{r_{i}+1+\eta_{i j}} \cdot \prod_{j=\varepsilon_{i}+1}^{\delta_{i}}\left(\gamma_{i n_{i}}-\gamma_{i j}\right)^{r_{i}+\eta_{i j}}\right]^{-1}
\end{aligned}
$$


We now estimate the denominator of $a_{(s k+l)(t k+h)}$.

Using the residue theorem we get:

$a_{(s k+l)(t k+h)}=\sum_{i=0}^{1} \sum_{j=0}^{1}((s-i) !(s-j) !)^{-1}$

$\cdot \sum_{m=\mu_{i j}}^{v_{i j} j} \sum_{m=\mu_{i j^{\prime}}}^{v_{i j^{\prime}}}\left(\frac{\partial^{(s-i)+(t-j)}}{\partial \xi_{1}^{(s-i)} \partial \xi_{2}^{(t-j)}}\left(\frac{f\left(\xi_{1}, \xi_{2}\right)}{\psi} \cdot\left(\xi_{1}-\alpha_{m}\right)^{s-i+1}\left(\xi_{2}-\beta_{n}\right)^{t-j+1}\right)\right)_{\xi_{1}=\alpha_{m} ; \xi_{2}=\beta_{n}}$

where $\mu_{00}=\mu_{00}^{\prime}=\mu_{10}^{\prime}=\mu_{01}=0, v_{00}=\mu_{10}-1=v_{01}=\mu_{11}-1=l, v_{00}^{\prime}=v_{10}^{\prime}$ $=\mu_{01}^{\prime}-1=\mu_{11}^{\prime}-1=h, v_{10}=v_{11}=\bar{k}-1$ and $v_{01}^{\prime}=v_{11}^{\prime}=k-1$.

Thus

$$
\begin{aligned}
& a_{(s k+l)(t k+h)}=\sum_{i=0}^{1} \sum_{j=0}^{1}((s-i) !(t-j) !)^{-1} \sum_{m=\mu_{i j}}^{v_{i}} \sum_{n=\mu_{i j}^{\prime}}^{v_{i j}^{\prime}} \sum_{x=0}^{s-i} \sum_{y=0}^{t-j}
\end{aligned}
$$

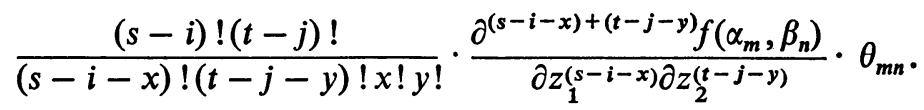

It is clear that $\left(\alpha_{m}-\alpha_{m}\right)=0$ and $\left(\beta_{n}-\beta_{n}\right)=0$ do not appear in this expression. Since

$$
\frac{\left(s+x_{j}\right) !}{s ! x_{j} !}, \frac{\left(t+y_{i}\right) !}{t ! y_{i} !}, \frac{\left(s+x_{j}-1\right) !}{(s-1) ! x_{j} !}, \frac{\left(t+y_{i}-1\right) !}{(t-1) ! y_{i} !}
$$

are integers for $j=0,1, \cdots, k-1$ and for $i=0,1, \cdots, k-1$ and since

$$
\frac{\partial^{\omega+q} f\left(\alpha_{m}, \beta_{n}\right)}{\partial z_{1}^{\omega} \partial z_{2}^{q}}
$$

are Gaussian integers for $\omega=0,1, \cdots ; q=0,1, \cdots ; m=0,1, \cdots, \bar{k}-1$ and for $n=0,1, \cdots, k-1$, we see that the denominator of the coefficient $a_{(s k+l)(t k+h)}$ divides the least common multiple of the quantities

$$
\begin{aligned}
(s-x) ! & \prod_{j=0 ; j \neq m}^{l}\left(\alpha_{m}-\alpha_{j}\right)^{s+1+x_{j}} \prod_{j=i+1 ; j \neq m}^{k-1}\left(\alpha_{m}-\alpha_{j}\right)^{s+x_{j}}(t-y) ! \\
& \prod_{i=0 ; i \neq n}^{h}\left(\beta_{n}-\beta_{i}\right)^{t+1+y_{i}} \prod_{i=h+1 ; i \neq n}^{k-1}\left(\beta_{n}-\beta_{i}\right)^{t+y_{i}}
\end{aligned}
$$

$m=0,1, \cdots, k-1 ; n=0,1, \cdots, k-1 ;\left\{x_{0}+x_{1}+\cdots+x_{k-1}=x, y_{0}+y_{1}+\cdots+y_{k-1}=y\right\} ;$ $x=0,1, \cdots, s$ and $y=0,1, \cdots, t$.

Let the least common multiple of these terms be

$$
b_{(s k+l)(t k+h)}=\prod_{p: \text { prime }} p^{\alpha_{p}} .
$$

Let us recall that $m_{1}=$ the maximum of the numbers $\left|\alpha_{m}-\alpha_{i}\right|$ as $m$ and $i$ run from 0 through $\vec{k}-1$ independently. 
If $|p|>m_{1}$ then $p$ can divide only $(s-x)$ ! among the factors

$$
(s-x) ! \prod_{j=0 ; j \neq m}^{l}\left(\alpha_{m}-\alpha_{j}\right)^{s+1+x_{j}} \prod_{j=l+1 ; j \neq m}^{k-1}\left(\alpha_{m}-\alpha_{j}\right)^{s+x_{j}}
$$

because $\left|\alpha_{m}-\alpha_{j}\right|<m_{1}<|p|$.

Now assume that $|p| \leqq m_{1}$.

Let $p^{\alpha}$ be the highest power of $p$ which appears among the set of all nonzero Gaussian integers whose absolute value $\leqq m_{1}$. Let $w$ be the largest (in absolute value) of these Gaussian integers divisible by $p^{\alpha}$ (in the sense that none of these Gaussian integers greater than $w$ are divisible by $p^{\alpha}$ ). Thus assume that $p^{\alpha} \cdot q=w$ or $|p|^{\alpha}|q|=|w|$ or

$$
\alpha=\frac{\log |w|}{\log |p|}-\frac{\log |q|}{\log |p|}
$$

and we know that this is an integer. Furthermore $|q|<|p|$, otherwise there would exist Gaussian integers of norm $\geqq|p|^{\alpha} \cdot|p|$. In particular, the Gaussian integer $p^{\alpha} p$ would be of norm $<m_{1}$ and, since it is divisible by $p^{\alpha+1}$, this contradicts the maximality of $\alpha$. Hence we get

$$
\alpha+\omega=\text { Integer }+\omega=\frac{\log |w|}{\log |p|},
$$

with $\omega<1$, so that

$$
\alpha=\left[\frac{\log |w|}{\log |p|}\right] \leqq\left[\frac{\log m_{1}}{\log |p|}\right]
$$

Note that we also have

$$
\frac{\log m_{1}}{\log |p|}<\frac{\log |w|}{\log |p|}+1
$$

otherwise we would get $m_{1} \geqq|w||p|$ which would imply that $w p$ is a Gaussian integer divisible by $p^{\alpha+1}$, contradicting the maximality of $\alpha$. However we shall not use this last fact. Hence we can maximize the power of $p$ which divides the product

$$
Q_{m}=(s-x) ! \prod_{j=0 ; j \neq m}^{l}\left(\alpha_{m}-\alpha_{j}\right)^{s+1+x_{j}} \prod_{j=l+1 ; j \neq m}^{k-1}\left(\alpha_{m}-\alpha_{j}\right)^{s+x_{j}}
$$

by setting $x_{i}=0$ for $i \neq j^{\prime}$ and $x_{j^{\prime}}=x$. Here $j^{\prime}$ is such that $p^{\alpha} \mid\left(\alpha_{m}-\alpha_{j}\right)$ implies that $p^{\alpha} \mid\left(\alpha_{m}-\alpha_{j^{\prime}}\right) j \neq m \neq j^{\prime}$. Thus we have for $|p| \leqq m_{1}$,

$$
p^{\theta_{m}} \mid(s-x) ! V\left[\alpha_{i}\right]^{s+1} p^{\left[\log m_{1} / \log p\right] x},
$$

where $p^{\theta_{m}}$ is the greatest power of $p$ which divides $Q_{m}$. 
Now we are interested in the highest powers of Gaussian primes which divide $(s-x)$ !. Since these powers will be multiplied by each other, we will be multiplying Gaussian primes by their conjugates. Therefore, in the final analysis, we may as well concern ourselves with the highest powers of the rational primes which divide $(s-x)$ !. We should note, however, that though we originally take all products over all Gaussian primes, we can take them over all rational primes because $a^{\alpha} \bar{a}^{\alpha}=(a \bar{a})^{\alpha}=p^{\alpha}$, where $a$ and, therefore, $\bar{a}$ and $p$ are primes, where $\bar{a}$ denotes the conjugate of $a$. Since $|\bar{a}|=|a|=p$ or $|\bar{a}|=|a|=p^{2}$ if $a=p$, the exponent which normally would be

$$
\frac{\log m_{1}}{\log |a|} \text { becomes } \leqq \frac{\log m_{1}}{\log p} .
$$

We now complete the proof using only rational primes.

Hence,

$$
\begin{aligned}
((s-x) !)_{p} & =\left[\frac{s-x}{p}\right]+\left[\frac{s-x}{p^{2}}\right]+\cdots=\frac{s-x}{p-1}+o(\log s) \\
& =(s !)_{p}-\frac{x}{p-1}+o(\log s) .
\end{aligned}
$$

$$
p^{\theta_{m}} \mid s ! V\left[\alpha_{i}\right]^{s+1} p^{\left(\left[\log m_{1} / \log p\right]-1 /(p-1)\right) x+o(\log s)}
$$

and similarly one gets an analogous expression for $\beta$. Thus it follows that

$$
\begin{aligned}
b_{(s k+l)(t k+h)} \mid s ! t !\left(V\left[\alpha_{i}\right]^{s+1} V\left[\beta_{i}\right]^{t+1}\right) \\
\cdot \prod_{p \leqq m_{1} ; p: \text { prime }} p^{\left(\left[\log m_{1} / \log p\right]-1 /(p-1)\right) s+o(\log s)} \\
\cdot \prod_{p \leqq m_{2} ; p: \text { prime }} p^{\left(\left[\log m_{2} / \log p\right]-1 /(p-1)\right) t+o(\log t)}
\end{aligned}
$$

Thus by virtue of (3.7) and (3.9) we get

$$
\begin{aligned}
\left|a_{(s k+l)(t k+h)} b_{(s k+l)(t k+h)}\right|< & {\left[\left(e \sigma_{1}+\varepsilon_{1}\right)^{s} s^{-s}\right] \cdot s^{s} \cdot e^{-s}\left|V\left[\alpha_{i}\right]^{s+1}\right| } \\
& \cdot \prod_{p \leqq m_{1} ; p: \text { prime }} p^{\left(\left[\log m_{1} / \log p\right]-1 /(p-1)\right) s} \\
& \cdot\left[\left(e \sigma_{2}+\varepsilon_{2}\right)^{t} t^{-t}\right] \cdot t^{t} \cdot e^{-t}\left|V\left[\beta_{i}\right]^{t+1}\right| \\
& \cdot \prod_{p \leqq m_{2} ; p \text { prime }} p^{\left(\left[\log m_{2} / \log p\right]-1 /(p-1)\right) t}
\end{aligned}
$$

and our theorem follows.

We now generalize Theorem 3.3.

In Theorem 3.3 we showed that for the functions discussed in that theorem

$$
\left|a_{s t}\right|<\left(\frac{e k+\varepsilon}{s}\right)^{s /(k+\varepsilon)}\left(\frac{e k+\varepsilon}{t}\right)^{t /(k+\varepsilon)}
$$


We note that when $s / t=\omega \rightarrow \infty$ as $s+t \rightarrow \infty$ we get:

$$
\begin{aligned}
\left|a_{s t}\right|< & \left(\frac{e \bar{k}+\varepsilon}{s}\right)^{t /(k+\varepsilon)}\left(\frac{e k+\varepsilon}{t}\right)^{t /(k+\varepsilon)}<\left(\frac{e \hat{k}+\varepsilon}{s}\right)^{s(k+\varepsilon)} \\
& \cdot\left(\frac{e k+\varepsilon}{s}\right)^{s / \omega(k+\varepsilon)} \omega^{s / \omega(k+\varepsilon)}<s^{-s /\left(k+\varepsilon^{\prime}\right)} s^{-s / \omega\left(k+\varepsilon^{\prime}\right)} \\
& \cdot \omega^{s / \omega(k+\varepsilon)}<s^{-s /\left(k+\varepsilon^{\prime}\right)} s^{-s / \omega\left(k+\varepsilon^{\prime}\right)} e^{s /(k+\varepsilon)} \\
= & s^{-s\left(1 /\left(k+\varepsilon^{\prime}\right)+1 / \omega\left(k+\varepsilon^{\prime}\right)\right)} e^{s /(k+\varepsilon)}<s^{-s /\left(k+\varepsilon^{\prime \prime}\right)} \\
< & \left(\frac{e \hat{k}+\varepsilon^{\prime \prime}}{s}\right)^{s /\left(k+\varepsilon^{\prime \prime \prime}\right)}\left(\frac{e k^{\prime}+\varepsilon^{\prime \prime \prime}}{t}\right)^{t /\left(k^{\prime}+\varepsilon^{\prime \prime \prime}\right)}
\end{aligned}
$$

for sufficiently large $s+t$, where $k^{\prime}$ is any number such that $0 \leqq k^{\prime}<k$. Thus Theorem 3.3 holds even with $k$ replaced by $k^{\prime}$ at least for the part dealing with order. A similar argument involving $\sigma_{1}$ and $\sigma_{2}$ can be argued so that we finally get the following theorem:

THEOREM 3.7. There exists a continuum of strongly transcendental entire functions with order point $\left(\rho_{1}, \rho_{2}\right)$ and type point $\left(\sigma_{1}, \sigma_{2}\right)$ which, together with all their derivatives, assume Gaussian integer values at the points

$$
\left(\alpha_{i}, \beta_{j}\right) ; i=1, \cdots, \bar{k} ; j=1, \cdots, k
$$

for any order point $\left(\rho_{1}, \rho_{2}\right)$ with $\rho_{1}>\bar{k}$ or with $\rho_{1}=\bar{k}$ and $\sigma_{1}>\sigma_{c 1}$.

A similar theorem holds with respect to $\rho_{2}$ and $\sigma_{2}$.

We now proceed with an analysis of order and type curves.

In the following we shall discuss the $k$-variable case. This can be accomplished with the same ease of notation as the 2-variable case. By the order set of $f$ we shall mean the set of all points on or above the order of $f$. We shall say that ord $f<$ ord $g$ iff order set of $f \subset$ order set of $g$.

To simplify our discussion we shall associate to the order set $\left\{\left(\rho_{1}, \cdots, \rho_{k}\right)\right\}$ of $f\left(z_{1}, \cdots, z_{k}\right)$, the set of points $\left\{\left(1 / \rho_{1}, \cdots, 1 / \rho_{k}\right)\right\}$ which we call the reciprocal order set of $f$.

THEOREM 3.8. The reciprocal order set of an entire function $f$, is convex.

Proof. Let $\left(a_{1}, a_{2}, \cdots, a_{k}\right)$ and $\left(b_{1}, b_{2}, \cdots, b_{k}\right)$ be any two points in the order set of $f$.

If $f=\sum a_{n_{1}} \cdots n_{k} z_{1}^{n_{1}} z_{2}^{n_{2}} \cdots z_{k}^{n_{k}}$, then we have

and

$$
\left|a_{n_{1} \ldots n_{k}}\right|<n_{1}^{-\left(n_{1} /\left(a_{1}+\varepsilon\right)\right)} n_{2}^{-\left(n_{2} /\left(a_{2}+\varepsilon\right)\right)} \cdots n_{k}^{-\left(n_{k} /\left(a_{k}+\varepsilon\right)\right)}
$$

$$
\left|a_{n_{1} \ldots n_{k}}\right|<n_{1}^{-\left(n_{1} /\left(b_{1}+\varepsilon\right)\right)} n_{2}^{-\left(n_{2} /\left(b_{2}+\varepsilon\right)\right)} \cdots n_{k}^{-\left(n_{k} /\left(b_{k}+\varepsilon\right)\right)}
$$

for sufficiently large $n_{1}+\cdots+n_{k}$. 
Thus for every $t(0 \leqq t \leqq 1)$ it follows that

where

$$
\begin{aligned}
& \left|a_{n_{1} \ldots n_{k}}\right|<n_{1}^{-n_{1}\left(t /\left(a_{1}+\varepsilon\right)+(1-t) /\left(b_{1}+\varepsilon\right)\right)} \cdots n_{k}^{-n_{k}\left(t /\left(a_{k}+\varepsilon\right)+(1-t) /\left(b_{k}+\varepsilon\right)\right)} \\
& <n_{1}^{-n_{1} /\left(c_{1}+\varepsilon^{\prime}\right)} \ldots n_{k}^{-n_{k} /\left(c_{k}+\varepsilon^{\prime}\right)}
\end{aligned}
$$

$$
1 / c_{i}=t / a_{i}+(1-t) / b_{i} \quad(i=1, \cdots, k)
$$

and our theorem follows.

THEOREM 3.9. For all $k$-tuples $\left(\alpha_{1}, \cdots, \alpha_{k}\right)$ with $\alpha_{i} \geqq 0$ for $i=1, \cdots, k$, there exists an entire function whose order is given by

$$
\alpha_{1} / \rho_{1}+\cdots+\alpha_{k} / \rho_{k}=1 ; \rho_{i}=0 \text { if } \alpha_{i}=0 .
$$

Proof. For the given $\alpha_{1}, \cdots, \alpha_{k}$, let

$$
f=\sum_{n=0}^{\infty}\left(z_{1}^{n_{1}} \cdots z_{k}^{n_{k}}\right) / n^{n}, n_{i}=\left[n \alpha_{i}\right] \quad(i=1, \cdots, k) .
$$

Here $f$ is independent of those $z_{i}$ for which $\alpha_{i}=0$ and hence $\rho_{i}=0$ for $\alpha_{i}=0$. We now restrict our attention to the positive $\alpha_{i}$. Since $a_{n_{1} \ldots n_{k}}=n^{-n}$ for $n_{i}=\left[n \alpha_{i}\right]$ and 0 otherwise the inequality

$$
a_{n_{1} \ldots n_{k}}<n_{1}^{-n_{1} /\left(\rho_{1}+\varepsilon\right)} \cdots n_{k}^{-n_{k} /\left(\rho_{k}+\varepsilon\right)}
$$

holds for sufficiently large $n_{1}+\cdots+n_{k}$ if and only if

$$
\begin{aligned}
& n^{-n}<\left[n \alpha_{1}\right]^{-\left[n \alpha_{1}\right] /\left(\rho_{1}+\varepsilon\right)} \ldots\left[n \alpha_{k}\right]^{-\left[n \alpha_{k}\right] /\left(\rho_{k}+\varepsilon\right)} \\
& =n^{-n\left(\alpha_{1} /\left(\rho_{1}+\varepsilon\right)+\ldots+\alpha_{k} /\left(\rho_{k}+\varepsilon\right)\right)} \cdot o\left(n^{c}\right) ;
\end{aligned}
$$

in other words if and only if

$$
\alpha_{1} / \rho_{1}+\cdots+\alpha_{k} / \rho_{k} \leqq 1 .
$$

THEOREM 3.10. Let $S$ be a convex set in the positive orthant of $E^{k}$ so that for every $\left(\sigma_{1}, \cdots, \sigma_{k}\right) \in S$ all points $\left(\sigma_{1}^{\prime}, \cdots, \sigma_{k}^{\prime}\right)$ with $0 \leqq \sigma_{i}{ }^{\prime} \leqq \sigma_{i}$ are in $S$. Then there exists an entire function $f$ whose reciprocal order set is $S$.

Proof. Let the points $p_{1}, p_{2} \cdots$ be a dense denumerable subset of points on $T$, the boundary of $S$ interior to the positive orthant. Let

$$
\alpha_{1 i} x_{1}+\cdots+\alpha_{k i} x_{k}=1
$$

be a supporting surface of $S$ going through $p_{i}$. As a result of our hypothesis on $S$ we have $\alpha_{j i} \geqq 0$.

By virtue of Theorem 3.9 there exists an entire function

$$
g_{i}=\sum_{n=0}^{\infty} n^{-n_{1}} z_{1}^{n_{1}} \cdots z_{k}^{n_{k}}, n_{i}=\left[n \alpha_{i}\right](i=1, \cdots, k)
$$

whose reciprocal order set is bounded by the plane (3.10) and the coordinate planes. 
For every $i$ choose $N_{i}$ such that

$$
\begin{aligned}
& \bar{g}_{i}=\sum_{n=N_{i}}^{\infty} n^{-n_{1} n_{1}} \cdots z_{k}^{n_{k}}<1 / 2^{i} \text { for }\left|z_{j}\right|<i, \\
& n_{j}=\left[n \alpha_{j i}\right] \quad(j=1, \cdots, k) \text {. } \\
& {\left[N_{i} \alpha_{1 i}\right]+\cdots+\left[N_{i} \alpha_{k i}\right]>i,} \\
& \left|a_{n_{1} \ldots n_{k}}^{i}\right|<n_{1}^{-n_{1} /\left(\rho_{1}+1 / i\right)} n_{2}^{-n_{2} /\left(\rho_{2}+1 / i\right)} \cdots n_{k}^{-n_{k} /\left(\rho_{k}+1 / i\right)}
\end{aligned}
$$

for every $\left(\rho_{1}^{-1}, \cdots, \rho_{k}^{-1}\right) \in S$, where $a_{n_{1} \ldots n_{k}}^{i}$ are the Taylor coefficients of $g_{i}$.

Let $g=\sum_{i=1}^{\infty} \bar{g}_{i}$. Clearly $g$ is entire and since all coefficients of $\bar{g}_{i}$ are nonnegative, the maximum of $|g|$ on the polycylinder is the sum of the maxima of the $\left|\bar{g}_{i}\right|$ so that ord $g>\operatorname{ord} \bar{g}_{i}$ for all $i$. In other words, the reciprocal order set of $g$ lies in the intersection of the reciprocal order sets of the $\bar{g}_{i}$ which is $S$. To prove that $S$ is the reciprocal order of $g$ we write

$$
g=\sum b_{n_{1} \ldots n_{k}} z_{1}^{n_{1}} \cdots z_{k}^{n_{k}},
$$

and it suffices to show that for any $\varepsilon>0$

$$
\left|b_{n_{1} \ldots n_{k}}\right|<n_{1}^{-n_{1} /\left(\rho_{1}+\varepsilon\right)} \cdots n_{k}^{-n_{k} /\left(\rho_{k}+\varepsilon\right)}
$$

for every $\left(\rho_{1}^{-1}, \cdots, \rho_{k}^{-1}\right) \in S$ and $n_{1}+\cdots+n_{k}$ sufficiently large. Choose $i_{0}$ such that $1 / i_{0}<\varepsilon / 2$ and $n_{1}+n_{2}+\cdots+n_{k}$ so large that

$$
\sum_{i=1}^{i_{0}-1}\left|a_{n_{1} \ldots n_{k}}\right|<n_{1}^{-n_{1} /\left(\rho_{1}+\varepsilon / 2\right)} \cdots n_{k}^{-n_{k} /\left(\rho_{k}+\varepsilon / 2\right)} \text {. }
$$

We note that

$$
\begin{aligned}
&\left|b_{n_{1} \ldots n_{k}}\right| \leqq \sum_{i=1}^{n_{1}+n_{2}+\ldots+n_{k}}\left|a_{n_{1} \ldots n_{k}}^{i}\right| \\
&= \sum_{i=1}^{i_{0}-1}\left|a_{n_{1} \ldots n_{k}}^{i}\right|+\sum_{i=i_{0}}^{n_{1}+n_{2}+\ldots+n_{k}}\left|a_{n_{1} \ldots n_{k}}^{i}\right|<n_{1}^{-n_{1} /\left(\rho_{1}+\varepsilon / 2\right)} \\
& \cdots n_{k}^{-n_{k} /\left(\rho_{k}+\varepsilon / 2\right)}+\left(n_{1}+n_{2}+\cdots+n_{k}\right) n_{1}^{-n_{1} /\left(\rho_{1}+1 / i_{0}\right)} \\
& \cdots n_{k}^{-n_{k} /\left(\rho_{k}+1 / i_{0}\right)}
\end{aligned}
$$

for $n_{1}+\cdots+n_{k}$ sufficiently large and our theorem follows.

By the type set of $f$ we shall mean the set of all points on or above the type of $f$. We now characterize types by proving the following two theorems.

THEOREM 3.11. Let $f$ be an entire function of order $\rho$. For every $\left(\rho_{1}, \cdots, \rho_{k}\right) \in \rho$ the type set $\sigma_{\rho_{1} \ldots \rho_{k}}=\left\{\left(\sigma_{1}, \cdots, \sigma_{k}\right)\right\}$ is such that $\left\{\left(\log \sigma_{1}, \cdots, \log \sigma_{k}\right)\right\}$ is convex.

Proof. Let $\left(\sigma_{1}, \cdots, \sigma_{k}\right)$ and $\left(\sigma_{1}^{\prime}, \cdots, \sigma_{k}^{\prime}\right)$ be any two type points in $\rho_{\rho_{1} \ldots \rho_{k}}$. We have for $f\left(z_{1}, \cdots, z_{k}\right)=\sum a_{n_{1} \ldots n_{k}} z_{1}^{n_{1}} \cdots z_{k}^{n_{k}}$ 
and

$$
\left|a_{n_{1} \ldots n_{k}}\right|<\left(e \rho_{1} \sigma_{1}+\varepsilon\right)^{n_{1} / \rho_{1}} \cdots\left(e \rho_{k} \sigma_{k}+\varepsilon\right)^{n_{k} / \rho_{k}} n_{1}^{-\left(n_{1} / \rho_{1}\right)} \cdots n_{k}^{-n_{k} / \rho_{k}}
$$

$$
\left|a_{n_{1} \ldots n_{k}}\right|<\left(e \rho_{1} \sigma_{1}^{\prime}+\varepsilon\right)^{n_{1} / \rho_{1}} \cdots\left(e \rho_{k} \sigma_{k}^{\prime}+\varepsilon\right)^{n_{k} / \rho_{k}} n_{1}^{-\left(n_{1} / \rho_{1}\right)} \cdots n_{k}^{-\left(n_{k} / \rho_{k}\right)} .
$$

Thus

$$
\left|a_{n_{1} \ldots n_{k}}\right|<\left(e \rho_{1} \sigma_{1}^{t} \sigma_{1}^{\prime(1-t)}+\varepsilon\right)^{n_{1} / \rho_{1}} \cdots\left(e \rho_{k} \sigma_{k}^{t} \sigma_{k}^{\prime(1-t)}+\varepsilon\right)^{n_{k} / \rho_{k}} n_{1}^{-\left(n_{1} / \rho_{1}\right)} \cdots n_{k}^{-\left(n_{k} / \rho_{k}\right)}
$$

for $0 \leqq t \leqq 1$.

Hence the point $\left(\sigma_{1}^{t} \sigma_{1}^{(1-t)}, \cdots, \sigma_{k}^{t} \sigma_{k}^{(1-t)}\right)$ is also in $\sigma_{\rho_{1} \ldots \rho_{k}}$. In other words if $\left(\log \sigma_{1}^{\prime}, \cdots, \log \sigma_{k}^{\prime}\right)$ and $\left(\log \sigma_{1}^{\prime \prime}, \cdots, \log \sigma_{k}^{\prime \prime}\right)$ are in $\left\{\left(\log \sigma_{1}, \cdots, \log \sigma_{k}\right)\right\}$ then $\left(t \log \sigma_{1}^{\prime}+(1-t) \log \sigma_{1}^{\prime \prime}, t \log \sigma_{2}^{\prime}+(1-t) \log \sigma_{2}^{\prime \prime}, \cdots, t \log \sigma_{k}^{\prime}+(1-t) \log \sigma_{k}^{\prime \prime}\right)$ is also in $\left\{\left(\log \sigma_{1}, \cdots, \log \sigma_{k}\right)\right\}$ and our theorem is proved.

REMARK. The same proof shows that if $\left(\sigma_{1}, \cdots, \sigma_{k}\right)$ is in the type set of $\left(\rho_{1}, \cdots, \rho_{k}\right)$ and $\left(\sigma_{1}^{\prime}, \cdots, \sigma_{k}^{\prime}\right)$ is in the type set of $\left(\rho_{1}^{\prime}, \cdots, \rho_{k}^{\prime}\right)$ then $\left(\sigma_{1}^{\prime \prime}, \cdots, \sigma_{k}^{\prime \prime}\right)$ is in the type set of $\left(\rho_{1}^{\prime \prime}, \cdots, \rho_{k}^{\prime \prime}\right)$ where $1 / \rho_{i}^{\prime \prime}=t / \rho_{i}+(1-t) / \rho_{i}^{\prime}$ and $\log \left(\rho_{i}^{\prime \prime} \sigma_{i}^{\prime \prime}\right) / \rho_{i}^{\prime \prime}=t \log \left(\rho_{i} \sigma_{i}\right) / \rho_{i}+(1-t)\left(\log \left(\rho_{i}^{\prime} \sigma_{i}^{\prime}\right) / \rho_{i}^{\prime}\right) 0 \leqq t \leqq 1$.

THEOREM 3.12. Given two $k$-tuples of positive numbers $\left(\rho_{1}, \cdots, \rho_{k}\right)$ and $\left(\alpha_{1}, \cdots, \alpha_{k}\right)$ and a set $\left\{\left(\sigma_{1}, \sigma_{2}, \cdots, \sigma_{k}\right)\right\}$ such that $\sigma_{i} \geqq 0$ for $i=1, \cdots, k$ and such that $\prod_{i=1}^{k} \sigma_{i}^{\alpha_{i} / \rho_{i}}$ $=$ constant $=K$, there exists an entire function with $\left(\rho_{1}, \cdots, \rho_{k}\right)$ as an order point and with type $\left\{\left(\sigma_{1}, \cdots, \sigma_{k}\right)\right\}$ at $\left(\rho_{1}, \cdots, \rho_{k}\right)$.

Proof. We assume that $\sum_{i=1}^{k} \alpha_{i} / \rho_{i}=1$. This condition is not necessary, but leads to a somewhat simpler proof. The function

$$
g=\sum_{n=1}^{\infty}\left(n^{-1} \prod_{i=1}^{k}\left(\rho_{i} / \alpha_{i}\right)^{\alpha_{i} / \rho_{i}} K e\right)^{n} z_{1}^{n_{1}} \cdots z_{k}^{n_{k}}, n_{i}=\left[n \alpha_{i}\right](i=1, \cdots, k)
$$

is entire and has the desired property.

It is clear from the proof of Theorem 3.9 that $g$ has $\left(\rho_{1}, \cdots, \rho_{k}\right)$ as an order point. Now let $\left(\sigma_{1}, \cdots, \sigma_{k}\right)$ be any type point of $g$ at $\left(\rho_{1}, \cdots, \rho_{k}\right)$. We get for sufficiently large $n$ that for any type point $\left(\sigma_{1}, \cdots, \sigma_{k}\right)$

$$
\left(\prod_{i=1}^{k}\left(\rho_{i} / \alpha_{i}\right)^{\alpha_{i} / \rho_{i}} K e\right)^{n} n^{-n}<\left(e \rho_{1}\left(\sigma_{1}+\varepsilon\right) / n \alpha_{1}\right)^{n \alpha_{i} / \rho_{i}} \ldots\left(e \rho_{k}\left(\sigma_{n}+\varepsilon\right) / n \alpha_{k}\right)^{n \alpha_{k} / \rho_{k}}
$$

or $K \leqq \prod_{i=1}^{k}\left(\sigma_{i}+\varepsilon\right)^{\alpha_{i} / \rho_{i}}$ for every $\varepsilon>0$ and conversely every point satisfying this inequality is a type point. Thus type is given by

$$
K=\prod_{i=1}^{k} \sigma_{i}^{\alpha_{i} / \rho_{i}}
$$

THEOREM 3.13. Let the set $\left\{\left(\log \sigma_{1}, \cdots, \log \sigma_{k}\right)\right\}$ which we denote by $\log \sigma$, be convex and such that all planes of support have outer normals in the positive orthant. Then $\sigma$ is the type set of some entire function $f$ at a prescribed order point $\left(\rho_{1}, \cdots, \rho_{n}\right)$ with $\rho_{i}>0$. 
Proof. Let the points $p_{1}, p_{2}, \cdots$ be a dense denumerable subset of points on the boundary of $\log \sigma$. For each $p_{i}$ we find a supporting plane $\alpha_{1 i} / \rho_{1} \log \sigma_{1}+\cdots+\alpha_{k i} / \rho_{k} \log \sigma_{k}=C_{i}$ where $\alpha_{j i}>0$ and $C_{i}$ is 1,0 , or -1 .

By virtue of Theorem 3.12, there exists a function

$$
g_{i}=\sum_{n=0}^{\infty}\left(n^{-1} \prod_{j=1}^{k}\left(\rho_{j} / \alpha_{j i}\right)^{\alpha_{j i} / \rho_{j}} K_{i}\right)^{n} z_{1}^{n_{1}} \cdots z_{k}^{n_{k}}, \quad K_{i}=e^{C_{i}},
$$

$n_{j}=\left[n \alpha_{j i}\right](j=1, \cdots, k)$ whose type at $\left(\rho_{1}, \cdots, \rho_{k}\right)$ is given by $\prod_{j=1}^{k} \sigma_{j}^{\alpha_{j i} / \rho_{j}}=K_{i}$ or by $\sum_{j=1}^{k} \alpha_{j i} / \rho_{j} \log \sigma_{j}=\log K_{i}$. For every $i$ choose $N_{i}$ such that

(a) $\bar{g}_{i}=\sum_{n=N_{i}}^{\infty}\left(n^{-1} \prod_{j=1}^{k}\left(\rho_{j} / \alpha_{j i}\right)^{\alpha_{j i} / \rho_{j}} K_{i}\right)^{n} z_{1}^{n_{1}} \cdots z_{k}^{n_{k}}<1 / 2^{i}, n_{j}=\left[n \alpha_{j i}\right](j=1, \cdots, k)$ for $\left|z_{t}\right|<i$ for $t=1, \cdots, k$.

(b) $\left[N_{i} \alpha_{1 i}\right]+\cdots+\left[N_{i} \alpha_{k i}\right]>i$,

(c) $\left|a_{n_{1} \ldots n_{k}}^{i}\right|<\left(\left(e \rho_{1} \sigma_{1}+1 / i\right) / n_{1}\right)^{n_{1} / \rho_{1}} \ldots\left(\left(e \rho_{k} \sigma_{k}+1 / i\right) / n_{k}^{n_{k} / \rho_{k}}\right)$ for every $\left(\sigma_{1}, \cdots, \sigma_{k}\right) \in \sigma$ for sufficiently large $n_{1}+n_{2}+\cdots+n_{k}$, where $a_{n_{1} \ldots n_{k}}^{i}$ is the Taylor coefficient of $g_{i}$.

Let $g=\sum_{i=1}^{\infty} \bar{g}_{i}$. Clearly $g$ is entire and its type at $\left(\rho_{1}, \cdots, \rho_{k}\right)$ is not below $\sigma$ at any point.

It suffices to show that for any $\varepsilon>0$ the Taylor coefficients $b_{n_{1} \ldots n_{k}}$ of $g$ satisfy

$$
\left|b_{n_{1} \ldots n_{k}}\right|<\left(\left(e \rho_{1} \sigma_{1}+\varepsilon\right) / n_{1}\right)^{n_{1} / \rho_{1}} \cdots\left(\left(e \rho_{k} \sigma_{k}+\varepsilon\right) / n_{k}\right)^{n_{k} / \rho_{k}} \text { for } n_{1}+\cdots+n_{k}
$$

sufficiently large for every $\left(\sigma_{1}, \cdots, \sigma_{k}\right)$ on $\sigma$. Choose $i_{0}$ such that $1 / i_{0}<\varepsilon / 2$ and $n_{1}+n_{2}+\cdots+n_{k}$ so large that

$$
\sum_{i=1}^{i_{0}-1}\left|a_{n_{1} \ldots n_{k}}^{i}\right|<\left(\left(e \rho_{1} \sigma_{1}+\varepsilon / 2\right) / n_{1}\right)^{n_{1} / \rho_{1}} \cdots\left(\left(e \rho_{k} \sigma_{k}+\varepsilon / 2\right) / n_{k}\right)^{n_{k} / \rho_{k}}
$$

We note that

$$
\begin{aligned}
& \left|b_{n_{1} \ldots n_{k}}\right| \leqq \sum_{i=1}^{n_{1}+\ldots+n_{k}}\left|a_{n_{1} \ldots n_{k}}^{i}\right|=\sum_{i=1}^{i_{0}-1}\left|a_{n_{1} \ldots n_{k}}^{i}\right|+\sum_{i=10}^{n_{1}+\ldots}\left|a_{n_{1} \ldots n_{k}}^{i+n_{k}}\right| \\
& <\left(\left(e \rho_{1} \sigma_{1}+\varepsilon / 2\right) / n_{1}\right)^{n_{1} / \rho_{1}} \cdots\left(\left(e \rho_{k} \sigma_{k}+\varepsilon / 2\right) / n_{k}\right)^{n_{k} / \rho_{k}} \\
& +\left(n_{1}+\cdots+n_{k}\right)\left(\left(e \rho_{1} \sigma_{1}+1 / i_{0}\right) n_{1}\right)^{n_{1} / \rho_{1}} \cdots\left(\left(e \rho_{k} \sigma_{k}+1 / i_{0}\right) / n_{k}\right)^{n_{k} / \rho_{k}} \text {. }
\end{aligned}
$$

This completes the proof of our theorem.

We now investigate entire functions with given order and type curves having integral derivatives at all points of a given rectangular array.

THEOREM 3.14. Let $g$ be an entire function with order curve $\rho$, where $\rho$ lies entirely in an infinite quadrant given by $\rho_{1}>a$ and $\rho_{2}>b$, with $(a, b)$ satisfying $a \geqq \bar{k}$ or $b \geqq k$. Then there exists a continuum of entire functions whose values and derivatives are integral at a given $\bar{k} \times k$ rectangular array of points and which have $\rho$ as their order curve. 
Sketch of Proof. By a method very similar to the proof of Theorem 3.3, we can choose an entire function $f$ such that

$$
\frac{\partial^{s_{h}+t_{l}}(f+g)\left(\alpha_{h}, \beta_{i}\right)}{\partial z_{1}^{s_{h} \partial z_{2}^{t_{l}}}}
$$

is a Gaussian integer sufficiently near to (contributions of terms preceding $a_{\text {st }}$ ), where all the terminology is like that in Theorem 3.3, with the understanding that

so that

$$
f=\sum_{s=0}^{\infty} \sum_{t=0}^{\infty} a_{s t}\left(z_{1}-\alpha_{1}\right)^{s_{1}} \cdots\left(z_{k}-\beta_{k}\right)^{t_{k}},
$$

$$
\left|a_{s t}\right| \leqq c\left|\left[s_{h} ! t_{l} !\left(\alpha_{h}-\alpha_{1}\right)^{s_{1}} \cdots\left(\beta_{k}-\beta_{i}\right)^{t_{k}}\right]\right|^{-1}
$$

for $c \geqq 1 / \sqrt{ } 2$, where $c$ is a function of the coefficients of $f$. Hence by an argument similar to that of Theorem 3.3 we can always find an $f$ such that

$$
\frac{\partial^{s_{h}+t_{l}}(f+g)\left(\alpha_{h}, \beta_{l}\right)}{\partial z_{1}^{s_{h}} \partial z_{2}^{t_{l}}}
$$

is a Gaussian integer and has order point $\left(\rho_{1}, \rho_{2}\right)$ for any $\left(\rho_{1}, \rho_{2}\right)$ such that $\rho_{1}>\bar{k}$ or $\rho_{2}>k$. Our theorem follows.

It is clear that with a similar proof we can show the following:

THEOREM 3.15. Let $f$ be an entire function with order point $\left(\rho_{1}, \rho_{2}\right)$ such that $\rho_{1}=k$. Let $\sigma$ be a type curve of $f$ at $\left(\rho_{1}, \rho_{2}\right)$ which is entirely contained in an infinite quadrant given by $\sigma_{1}>a$ and $\sigma_{2}>b$, where $a \geqq \sigma_{c 1}$ or $b \geqq \sigma_{c 2}$ and where

$$
\sigma_{c 1}=\left|V\left[\alpha_{i}\right]\right|^{-2 / k} \text { and } \sigma_{c 2}=\left|V\left[\beta_{i}\right]\right|^{-2 / k} \text {. }
$$

Then there exists a continuum of entire functions whose values and derivatives are Gaussian integers at a given $k \times k$ rectangular array of points which have $\sigma$ as their type curve at $\left(\rho_{1}, \rho_{2}\right)$.

It is also natural to conjecture that if for a given array of pairs of integers, for every point on a hyperbolic convex curve $\phi$, there exists an entire function with its value and derivatives integral at the given array and which has this point as an order point, then there exists an entire function, whose value and derivatives are integral at the given array and which has $\phi$ as its order curve.

\section{BIBLIOGRAPHY}

1. E. G. Straus, On entire functions with algebraic derivatives at certain algebraic points Ann. of Math. 52 (1950), 188-198.

2. - On the polynomials whose derivatives have integral values at the integers, Proc. Amer. Math. Soc. 2 (1951), 24-27.

3. T. Schneider, Ein satz über ganzwertige funktionen als prinzip für transzendentbeweise, Math. Ann. 121 (1948), 131-140. (See other references in this paper.) 
4. S. Kakeya, Notes on the maximum modulus of a function, Tôhoku Math. J.10(1916), 68-70.

5. G. Pólya, Über die kleinsten ganzen funktionen deren samtliche derivierte im punkte $z=0$ ganzzahlig sind, Tôhoku Math. J. 19 (1921), 65-68.

6. L. Bieberbach, Über einen satz Pólyascher Art, Arch. Math. 4 (1953), 23-27,

7. - - Theorie der geometrischen Konstruktionen, Lehrbücher and Monographen aus dem Gebiete der exakten Wissenschaften, Bd. 13, Verlag Birkhäuser, Basel, 1952.

8. D. Sato and E. G. Straus, On the rate of growth of Hurwitz entire functions, J. Math. Soc. Japan 1962 based on dissertation, University of California, Los Angeles, 1961.

9. B. A. Fuks, Theory of analytic functions of several complex variables, Math. Mono., Vol. 8, Amer. Math. Soc., Providence, R. I., 1963, 374 pp.

NAVAL Research Laboratory,

WASHINGTON, D.C. 